\title{
Business Intelligence in Healthcare Industry
}

\author{
Anmol Khanna ${ }^{1}$, Dhruv Bhasin ${ }^{2}$ \\ ${ }^{1,2}$ The North Cap University, School of Engineering and Technology, Huda Sector 23A, Gurgaon 122017, Haryana, India
}

\begin{abstract}
Business intelligence is a collection of decision support technologies for the enterprise aimed at enabling knowledge workers such as professionals to make better and faster decisions. Interest in implementation of business intelligence (BI) technologies in different sectors has been increasing from year to year. Recently it has found its tremendous demand in healthcare industry. There are advanced technologies that provide efficient methods and appropriate environment for implementation of BI in the healthcare sector. $B I$ has a very important role in the healthcare industry due to the availability of data in diverse formats which makes its access difficult across different departments (For e.g. Some data regarding the customer details is with the admin dept. and some reports of test are with the concerned lab), this makes difficult to assemble and interpret data, but due to robust environment BI tools provide, the task has been made easy to a great extent.
\end{abstract}

Keywords: Business Intelligence, Healthcare, Data Warehouse, Data Mining.

\section{Introduction}

Business intelligence, or $\mathrm{BI}$, is a term that is being thrown around incessantly in the business world today. It is a technology-driven process for analyzing data. It helps in presenting actionable information to help corporate executives, managers and other end users make more informed business decisions. Tools that are available in BI make it possible for organization to understand the market trends and make suitable decisions.

A Data Warehouse is a consolidation of data from a variety of sources designed to support strategic and tactical decision making. Its purpose is to provide a coherent picture of the business at a point in time. Using various DM toolsets, users are able to execute online queries and 'mine' their data.

Many successful companies have been investing sums of money in business intelligence and data warehousing tools and technologies as a result of which the organizations have been able to have an updated version of data of their patients and the suppliers. E.g. Blue Health intelligent Database started in 2006.

The potential benefits of business intelligence programs include accelerating and improving decision making; optimizing internal business processes; increasing operational efficiency; driving new revenues; and gaining competitive advantages over business opponents. The systems can also help companies identify market trends and spot business problems that need to be addressed.

BI data can include past information, as well as new data gathered from source systems as it is being generated, allowing BI analysis to support both strategic and tactical decision-making processes. Originally, BI tools were primarily used by data analysts and other IT professionals who ran analyses and produced reports with query results for business users. Business executives and workers are using BI software themselves, partly to the development of selfservice BI and data discovery tools.

Just like the advancement in volume of data in various sectors like the Ecommerce, data in healthcare sector has also been increasing rapidly. Data is being generated from genotyping, gene data and from electronic health records, insurance records, feedback etc. Moreover, healthcare is one of the fundamental tasks of modern states, and issues relating to them are the subject of interest in various sectors. The transformation of the healthcare sector has therefore become the recent subject of research in many fields, especially medicine, psychology, social policy. Recently, it has become the subject of interest for economists, as well as management and IT specialists.

Nowadays, healthcare systems face challenges due to changes in attitudes of people towards health, technological advances in medicine and the limited possibilities to increase health funding, thus requiring more intensive search for the effectiveness of the systems. The impact on this state of affairs has a lot of factors, which include: rising costs of medical services, rapid development of medical technologies, increasing aging population, changing disease patterns, rising expectations and entitlements of patients, large-scale inefficiencies in health care, the pressure to increase access to healthcare, the growing diversity of medical services, changes in the approach to financing and managing of healthcare and the growing importance of information technology in the sector.

Due to the above mentioned reasons we have been able to see the increasing gap between the healthcare needs and the funds to meet these demands; hence the only feasible solution is the implementation of IT for data access and enabling faster and efficient decision making. 


\section{International Journal of Science and Research (IJSR) \\ ISSN (Online): 2319-7064}

Index Copernicus Value (2013): 6.14 | Impact Factor (2014): 5.611

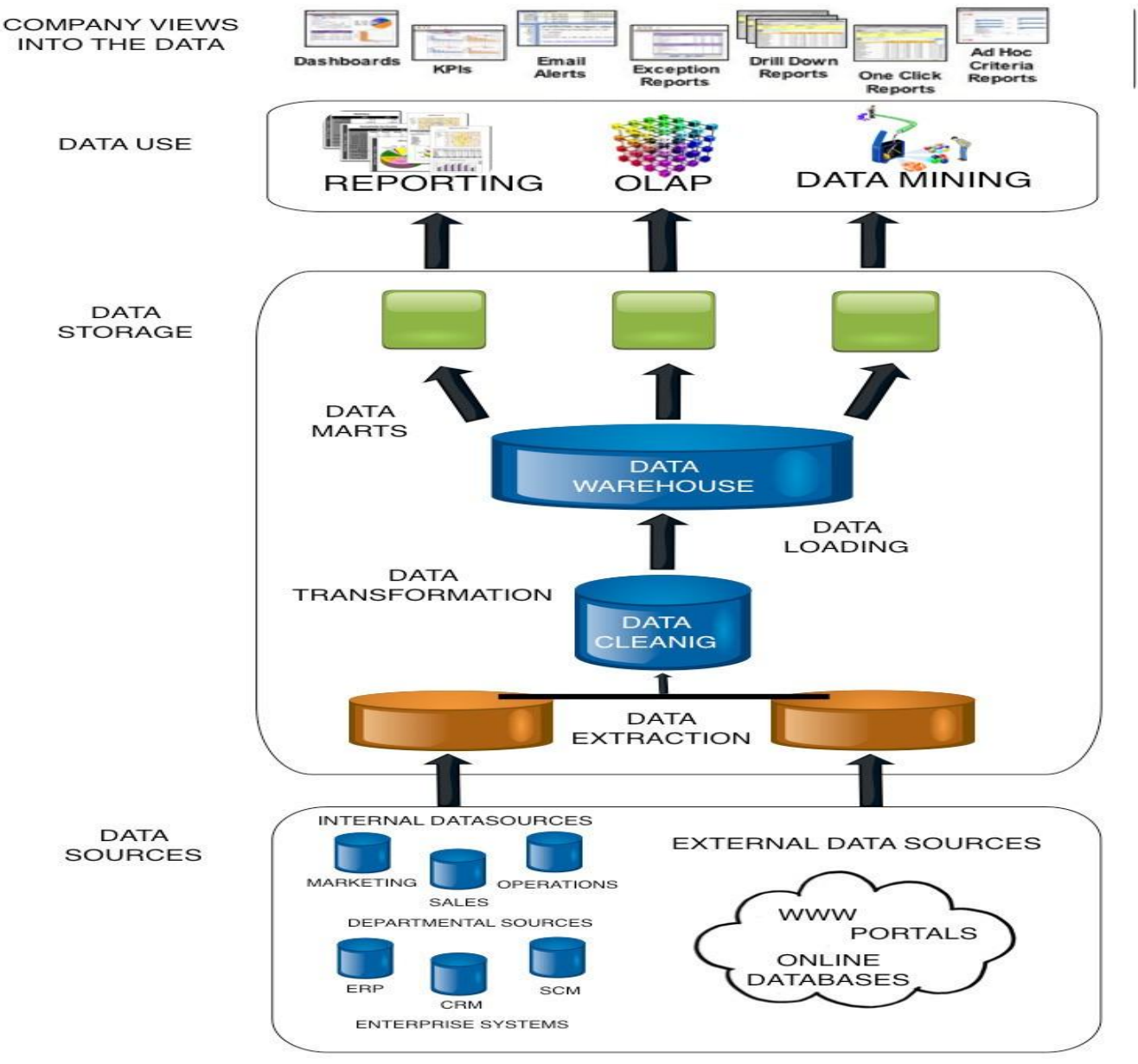

Figure 1: Showing a BI system

\section{Current Scenario}

Healthcare activities include providing preventive measures, mitigation and treatment to ensure good health for the people. Healthcare as a system is a group of individual doctors and institutions (Hospitals) aimed at providing public healthcare. Health is considered as a value which can't be purchased while the medical services (consultation) and goods (medicines) can be purchased to ensure good health.

Though market for medical services exist but it is characterized by availability of inconsistent information i.e. patients don't have sufficient knowledge about their condition, hence have not been able to make rational choices and purely depend upon the expertise of the doctor and therefore the organizations should provide information to customers about the medical services and its effectiveness which will only be possible if the organization has past history about the patient.

The current model of health system consist of three elements service seekers (patients), providers (doctors) and payers (insurer). The most important feature of healthcare is the wide variety in the way (how), place (where) and time (when) to provide services to the patient. This has resulted in the introduction of the division into: primary healthcare and specialized, outpatient care (open) and stationary (closed), emergency care, short and long term treatment. The only way to improve the database has been to make best use of the available databases, existing patient data and the financial data.

Few of the shortcomings in the healthcare system:

- Poor quality of health information - redundancy, inconsistent standards for the collection and sharing of information,

- Inability to obtain health information at the time and place where it is needed - the data collected in health records are limited, and EHR (Electronic Health Record) data are never collected. Some registers in health exist only in paper form, that limits the quick access to them,

- Inadequate procedures - implementation of information systems not related to the relevant organizational changes,

In the existing system the data has been available across different departments and across various Vendors (Laboratories) and these never communicate with each other hence leading to data inconsistency and redundancy.

Hence keeping in mind the current scenario and the Financial constraints the best bet is to adapt to $\mathrm{BI}$ technologies and start operating on data warehouses, data marts containing critical information like patient history, invoices, data regarding occupancy in the hospitals and radiology images and scanned documents. This step has been becoming very common in today's mobility world and one of the organizations namely Dr. Lal path lab has shifted on to these strategies and the reports are wirelessly delivered onto your

\section{Volume 4 Issue 12, December 2015}




\section{International Journal of Science and Research (IJSR) \\ ISSN (Online): 2319-7064}

Index Copernicus Value (2013): 6.14 | Impact Factor (2014): 5.611

mails and shared with the concerned doctors eliminating all the physical work that used to be needed previously.

\section{BI in Health Care}

One of solutions to this problem has been healthcare information technology (HIT), including tools for efficient and effective maintenance and sharing of data. These systems are based on integrated management systems for supporting the holistic management of business organizations (Enterprise Resource Planning, ERP). And even in the medical facilities classic ERP systems adjustable for medical activities (e.g., Impuls 5 BPSC) are implemented. All these systems have modular structure. Most professional healthcare organizations all over the world now rely on Healthcare (Hospital) Information Systems (HIS) that help them manage all their medical and administrative information and using Healthcare Information System or Patient Data Management System (PDMS). All these terms refer to the integrated information systems that have been used to manage organizations in the healthcare sector. They are a complete solution for managing the medical, administrative, financial and private data. The main aim of HIS has been to provide support patient care, achieving financial targets and make administration efficient.

HIS consists of tools that cater to needs of all the users and the departments within the organization and they help in efficient management of data from patient private information to financial data, previous medical history, various images and reports of XRAYS etc. Having a well developed HIS provides numerous benefits like better treatment and care. EMR (electronic medical record) might pose as a record for patient in a particular department and act as a component for the EHR. EHR is maintained by most hospitals and clinics thus providing doctors and the patients all the relevant information regarding the patient and the previous medical history allowing for better and efficient treatment and medical services. EIS (executive information systems) provides data in a structured format allowing for easy and efficient access to data from various sources.

Hence the fundamental reasons for adapting BI methodology are:

- It allows the users as well as employees of the organization to get detailed records allowing to make quicker and efficient decisions i.e. information is available under a single source hence there is no need to worry about the data inconsistency or data redundancy etc.

- They allow complex data from various sources to be structured into an understandable format i.e. data in form of XRAY images, scanned reports; prescriptions etc are stored together allowing user and the employee to gain knowledge about past history easily.

- BI can be used to make strategic decisions regarding profit making: As organization has the complete data of its operations and financial records, it can easily make decisions about where to cut the costs and how to improve facilities based on customer feedback allowing generating more profit for the organization.
- Provide access to data on multiple platforms and various departments: As data has been made available under a single source, so the organization allow users to access the data in various devices like tablets, desktops through cloud storage.

- They allow organization to work in teams: It enables the organization to divide its work force into small teams and assigning a particular department to each team along with the relevant data allowing making effective decisions.

- Theses help in adapting to new technologies fast and in an effective manner: Adapting and implementing new piece of technology has been made easier by the fact that all relevent data is stored together.

- Enables organization to be flexible.



Figure 2: Showing BI in a healthcare organization

\section{Potential Challenges for Application of BI}

- Availability of funds: shifting to such a complex technology requires a lot of initial funding.

- Lack of resources: Such a complex system requires availability of equipments like server, fast computing machines but availability of these items might be a constraint.

- Complexity of IT Equipments: Healthcare organization has many complex machines e.g. one for radiology, other for XRAY etc. so combining and studying data from such complex machines might pose a big challenge for the analysts.

- Future Scope: As everyone knows technology keeps on changing practically on a yearly basis, so the organization has to decide if it's feasible enough to make such an investment.

- Lack of knowledge among the staff members: Using such an advanced technology requires the availability of the knowledgeable personnel and those who can understand and help in making effective decisions.

- Identification of useful information: As data is unstructured and can come from a variety of sources so it might be difficult to segregate between relevant and irrelevant data.

- Data Standards: Organizations need to have a standard according to which such complex data can be structured. 


\section{International Journal of Science and Research (IJSR) \\ ISSN (Online): 2319-7064}

Index Copernicus Value (2013): 6.14 | Impact Factor (2014): 5.611

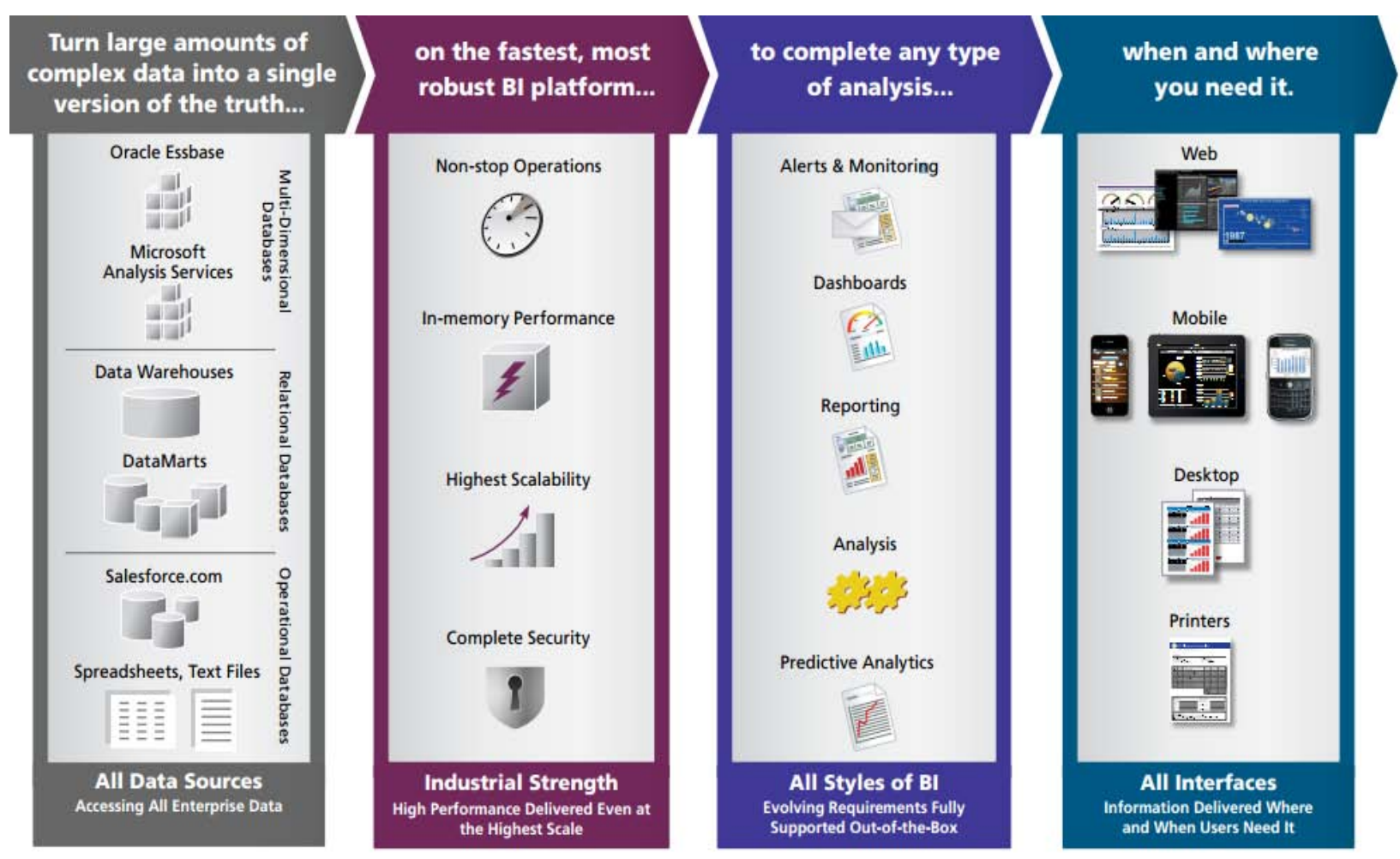

Figure 3: Showing the application of BI in Healthcare Industry

\section{References}

[1] http://www.microstrategy.com/Strategy/media/download s/solutions/MicroStrategy-Mobile-Healthcare-ProvidersBrochure.pdf:

[2] https://www.healthcatalyst.com/healthcare-businessintelligence-data-warehouse

[3] http://www.informationweek.com/administrationsystems/11-bi-tools-to-analyze

[4] Data Mining: Concepts and Techniques, Han,Jiawei

\section{Author Profile}

Anmol Khanna received the B.Tech degree in Computer Science Engineering from The North Cap University in 2015.

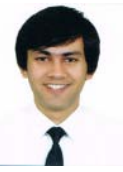

Dhruv Bhasin received the B.Tech degree in Information Technology Engineering from The North Cap University in 2015. 